\title{
BMJ Open Digital technology and disease surveillance in the COVID-19 pandemic: a scoping review protocol
}

\author{
Lorie Donelle, ${ }^{1}$ Jodi Hall, ${ }^{1,2}$ Brad Hiebert, ${ }^{1}$ Jacob J Shelley, ${ }^{3,4}$ Maxwell J Smith, ${ }^{4}$ \\ Jason Gilliland, ${ }^{4,5,6}$ Saverio Stranges, ${ }^{6,7,8,9}$ Anita Kothari (D) , ${ }^{4}$ Jacquelyn Burkell, ${ }^{10}$ \\ Tommy Cooke, ${ }^{11}$ Jed Long, ${ }^{5}$ James M Shelley, ${ }^{1}$ Deanna Befus, ${ }^{1}$ Leigha Comer (D),${ }^{1}$ \\ Marionette Ngole, ${ }^{1}$ Meagan Stanley ${ }^{12}$
}

To cite: Donelle L, Hall J, Hiebert B, et al. Digital technology and disease surveillance in the COVID-19 pandemic: a scoping review protocol. BMJ Open 2021;11:e053962. doi:10.1136/ bmjopen-2021-053962

- Prepublication history and additional supplemental material for this paper are available online. To view these files, please visit the journal online (http://dx.doi.org/10.1136/ bmjopen-2021-053962).

Received 31 May 2021 Accepted 08 0ctober 202
Check for updates

(C) Author(s) (or their employer(s)) 2021. Re-use permitted under CC BY-NC. No commercial re-use. See rights and permissions. Published by BMJ.

For numbered affiliations see end of article.

Correspondence to

Dr Lorie Donelle;

Idonelle@uwo.ca

\section{ABSTRACT}

Introduction Infectious diseases pose a risk to public health, requiring efficient strategies for disease prevention. Digital health surveillance technologies provide new opportunities to enhance disease prevention, detection, tracking, reporting and analysis. However, in addition to concerns regarding the effectiveness of these technologies in meeting public health goals, there are also concerns regarding the ethics, legality, safety and sustainability of digital surveillance technologies. This scoping review examines the literature on digital surveillance for public health purposes during the COVID-19 pandemic to identify health-related applications of digital surveillance technologies, and to highlight discussions of the implications of these technologies.

Methods and analysis The scoping review will be guided by the framework proposed by Arksey and O'Malley and the guidelines outlined by Colquhoun et al and Levac et al. We will search Medline (Ovid), Psyclnfo, PubMed, Scopus, CINAHL (EBSCOhost), ACM Digital Library, Google Scholar and IEEE Explore for relevant studies published between December 2019 and December 2020. The review will also include grey literature. Data will be managed and analysed through an extraction table and thematic analysis.

Ethics and dissemination Findings will be disseminated through traditional academic channels, as well as social media channels and research briefs and infographics. We will target our dissemination to provincial and federal public health organisations, as well as technology companies and community-based organisations managing the public response to the COVID-19 pandemic.

\section{INTRODUCTION}

The COVID-19 pandemic constitutes an unparalleled global crisis impacting all matters that determine health (eg, environment, economy, health services) and has been described as the first pandemic of 'the algorithmic age' where advanced data analytics are contributing to sophisticated detection, treatment and prevention strategies. ${ }^{1}$ Bennet et $a t^{2}$ describe surveillance practices as central to all organisations and sectors, and encourage attentiveness to
Strengths and limitations of this study

- This scoping review will highlight existing evidence of digital surveillance strategies for disease mitigation used during the COVID-19 pandemic and identify any gaps in the literature related to technology type, design, and implementation of digital surveillance strategies, and implications related to data ownership, privacy and the sustainability of these initiatives.

- Our focus on the global context will allow us to compare uses of digital health surveillance technology across regions and nations.

- Our search of the literature draws on a wide variety of databases and employs a broad understanding of digital health surveillance technology.

- Our focus is on digital health surveillance technology used during the COVID-19 pandemic, which may limit our ability to investigate forms of digital surveillance used during previous pandemics, epidemics and outbreaks. While we initially intended to review publications from 2000 to 2020 to trace the use of digital health surveillance technologies over time and during different outbreaks, our literature search yielded an unmanageable number of results that we could not review with our available time and resources. As such, this review has been limited to surveillance technologies used during the COVID-19 pandemic. While this limit in scope will exclude the opportunity to analyse developments in the use of digital health surveillance technology over time, our team of researchers found that limiting the scope to the COVID-19 pandemic was the most effective means of retaining a manageable number of publications for review while also answering our modified research questions.

- Including only publications written in English will exclude discussions and analyses of digital health surveillance technology in other languages, which may limit our capacity to take a global approach.

misuse of data collected for another purpose: function or mission creep. ${ }^{2}$ Defined as "the focused, systematic, and routine attention 
to personal details for purposes of influence, management, protection or direction', ${ }^{3}$ surveillance constitutes a long-standing practice within public health. There has been a surge in digital surveillance technology development by academics, private sector companies and 'citizen scientists' to support public health practices (eg, contact tracing, physical distancing). ${ }^{14}$

The use of existing digital surveillance technologies has also been leveraged and redirected to support pandemic management. ${ }^{5}$ To date, the use of technology to mitigate the spread of COVID-19 within and across countries has achieved varying levels of success, dependent on indicators of success (eg, disease containment (testing, vaccinations), mortality, educational/school attendance, employment rate, real gross domestic product), which vary geographically. Globally, governments are considering, or are currently using, digital surveillance technologies (eg, cellphone geolocation, closed-circuit cameras, apps) and mass public data collection (eg, wastewater surveillance) to detect and mitigate the spread of the COVID-19 virus, and to ensure compliance with public health measures. ${ }^{6}$

There have also been concerns about (mis) uses of digital technology measures during pandemic and non-pandemic situations. Many have voiced concerns regarding the short-term and long-term potentials of these technologies, including undermining human rights, ${ }^{7}$ threatening our fundamental values, ${ }^{89}$ inequitable targeting of oppressed and racialised communities, ${ }^{10}$ biases embedded in coding leading to discriminatory practices, ${ }^{11-13}$ inequitable power structures ${ }^{14}$ and engendering a false sense of security. ${ }^{15}$ Researchers, human rights advocates and knowledge leaders in digital technology are insistent that governments and healthcare decision-makers balance technological innovation as a pandemic response with transparency, diligence and attentiveness to issues of data standards, ethics, equity and human rights to effectively address the short-term and long-term implications on health and issues that determine health. ${ }^{16}$ Patel,${ }^{17}$ for instance, argues that:

while data can save lives at times of global public health crisis...it can only do this effectively if its use, management and governance, even at times of crisis, is underpinned by clear rules (grounded in law, ethics and human rights) about how best to use data; and trust in institutions to use data well.

However, the urgency to control the spread of COVID-19 has effectively limited opportunities to thoroughly consider the intended (disease containment) and unintended (eg, violation of ethical practices and human rights standards) consequences. ${ }^{17}$ Digital surveillance technologies that bear on determinants of health require regulatory oversight that accounts for transparency, diversity, networks of control, influence and the potential for the exploitation of citizen data by public and private organisations. $^{1819}$
This scoping review aims to investigate the peer-reviewed and grey literature on the use of digital surveillance technologies for public health mitigation purposes during the COVID-19 pandemic and within the global context. The objectives of the scoping review are as follows:

- To review the breadth and depth of the academic and grey literature on digital health surveillance technologies and their use during the COVID-19 pandemic.

- To explore how the literature has taken up and addressed the short-term and long-term implications of digital surveillance technology on diverse populations, particularly those who are marginalised or facing existing inequities.

- To identify gaps in the peer-reviewed and grey literature.

\section{METHODS AND ANALYSIS}

We will conduct a scoping review with guidance from Arksey and O'Malley, ${ }^{20}$ Colquhoun et al, ${ }^{21}$ Levac et al, ${ }^{22}$ and the Joanna Briggs Institute guidelines. ${ }^{20-23}$ A scoping review was determined to be the most appropriate means of addressing our research objectives, as our intent is to explore what is known about digital surveillance technologies for public health purposes and to investigate the state of the literature. To this end, we look to use a scoping strategy to map relevant literature in the field of interest. ${ }^{20}$ Our aim is to convey the breadth and depth of the peer-reviewed and grey literature on this topic. ${ }^{21}$ We will also trace these various forms of investigation and discussions to identify any gaps that might exist.

This scoping review will follow the methodological framework described by Arksey and O'Malley, ${ }^{20}$ which comprises five stages: (1) identifying the research question, (2) identifying relevant studies, (3) study selection, (4) charting the data, (5) collating, summarising and reporting the results. ${ }^{20}$ In writing this scoping review protocol, we also drew on the Preferred Reporting Items for Systematic Review and Meta-Analysis (PRISMA) Protocols reporting guidelines. ${ }^{24}$

\section{Stage 1: identifying the research question}

Our scoping review will be guided by the following research question: What is known about digital health surveillance technologies targeted at citizen surveillance during the COVID-19 pandemic within the global context? In addition to this research question, we also seek to answer the following subquestions: (1) What are the health-related applications of digital surveillance technology strategies? (2) What are the existing and/or predicted short-term and long-term implications of digital surveillance technology on diverse cultural, criminalised, Indigenous, disabled and otherwise marginalised populations?

\section{Stage 2: identifying relevant literature}

Our interdisciplinary team of researchers informed the adoption of an expansive definition of digital health surveillance technologies that includes any use of technology with the goal of making someone, or something, visible 
Box 1 Search strategy and search terms developed in consultation with the research librarian
1. Population Surveillance/or Public Health Surveillance/or surveil- lance.tw.
2. digital surveillance.tw.
3. biosurveillance.tw. or Biosurveillance/
4. epidemiological monitoring.tw. or Epidemiological monitoring/
5. 1 or 2 or 3 or 4
6. pandemic.tw. or Pandemics/
7. disease outbreak.tw. or Disease Outbreaks/
8. Coronavirus Infections/or covid-19.tw.
9. covid19.tw.
10. H1N1.tw.
11. SARS.tw. or SARS Virus/
12. 6 or 7 or 8 or 9 or 10 or 11
13. Public Health/or public health application.mp
14. 5 and 12

for public health purposes. We developed our search strategy through ongoing consultations with a specialist subject librarian, who assisted in developing the search strategy and identifying relevant databases. The search strategy will include pertinent and comprehensive search terms that represent the primary concepts of this scoping review's objectives. These consist of keywords and medical subject headings (MeSH) terms, as well as combinations of these terms using Boolean operators (box 1). The search strategy and keywords will be adjusted for each database (see online supplemental file 1).

An electronic search will be conducted using the following databases: Medline (Ovid), PsycInfo (Ovid), PubMed, Scopus, CINAHL, ACM Digital Library, Google Scholar and IEEE Explore. The databases were chosen with the intention of including perspectives from health, public health, engineering, computer science, data ethics and other specialist fields on the use of technology for health surveillance purposes. We will also hand search key journals and the reference lists of relevant articles for additional publications that may have been missed from the database searches. All references will be exported to a reference manager software to organise references and remove duplicates.

Grey literature from organisations with relevance to the focus of our research (eg, digital health, surveillance, data/human rights, ethics, equity, privacy) will be included. With the help of a research librarian, our team of interdisciplinary researchers selected relevant organisational websites that explore the use and applications of digital technology for surveillance purposes. We will conduct a search of these websites to retrieve potentially relevant grey literature. These sites include: the Canadian Agencies for Drugs and Technology in Health, the Ada Lovelace Institute, the Center for International Governance Innovation, the Geneva Internet Platform, Munk Updates, Human Rights Watch, the International Civil Liberties Monitoring Group, the Surveillance Studies Centre at Queen's University, the Information and Privacy
Commissioner of Ontario, Privacy International, Amnesty International, the International Association of Privacy Professionals, PreventionWeb, the National Health Policy Forum and the Mitre.

These websites will be searched through a manual search of current and archived contents and, where applicable, through the use of the internal search tool on each website. We will use similar key terms to those being used to search the peer-reviewed literature. Any relevant literature published between December 2019 and December 2020 will be retained for further review. Links to potentially relevant publications will be extracted to a spreadsheet for further screening by two researchers.

\section{Stage 3: literature selection \\ Inclusion criteria}

We began with a broad search of the literature to capture all publications on the use of digital health surveillance technology during pandemics, epidemics and outbreaks published between January 2000 and December 2020. As we are interested in the global use of digital health surveillance technologies, we included publications written from, and about, all countries and regions. However, due to limitations in time and resources, we only included publications written in English. This search yielded 9630 results. From these results, we screened the abstracts based on the following inclusion criteria:

- The publication must include mention of the use of a digital technology for public health surveillance.

- This public health surveillance must be oriented towards the containment or mitigation of the spread of an infectious disease.

- Public health surveillance through digital technology must be focused on surveilling humans, not nonhuman animals.

After screening the abstracts, we retained 2076 publications for inclusion. Next, we read each publication to screen against the inclusion criteria listed above. Following this screening process, we retained 888 publications for review.

Given the resources and time available to us, it was impractical to attempt a scoping review of over 800 publications. Our research team trialled several ways of further limiting our scope as a means of reducing this number. We experimented with limiting the scope by technology, by region, by methodology and according to whether the technology was publicly or privately funded, but these exclusions either limited the scope in such a way that we could not answer our research questions or were ineffective at reducing the number of included publications to a manageable amount.

We next attempted to limit the scope to focusing solely on digital health surveillance technologies used during the COVID-19 pandemic. We refined our inclusion criteria to limit the publication timeframe from December 2019 to December 2020, and we excluded publications that did not have the terms 'coronavirus', 'COVID19', 'SARSCoV-2' or 'severe acute respiratory syndrome coronavirus 


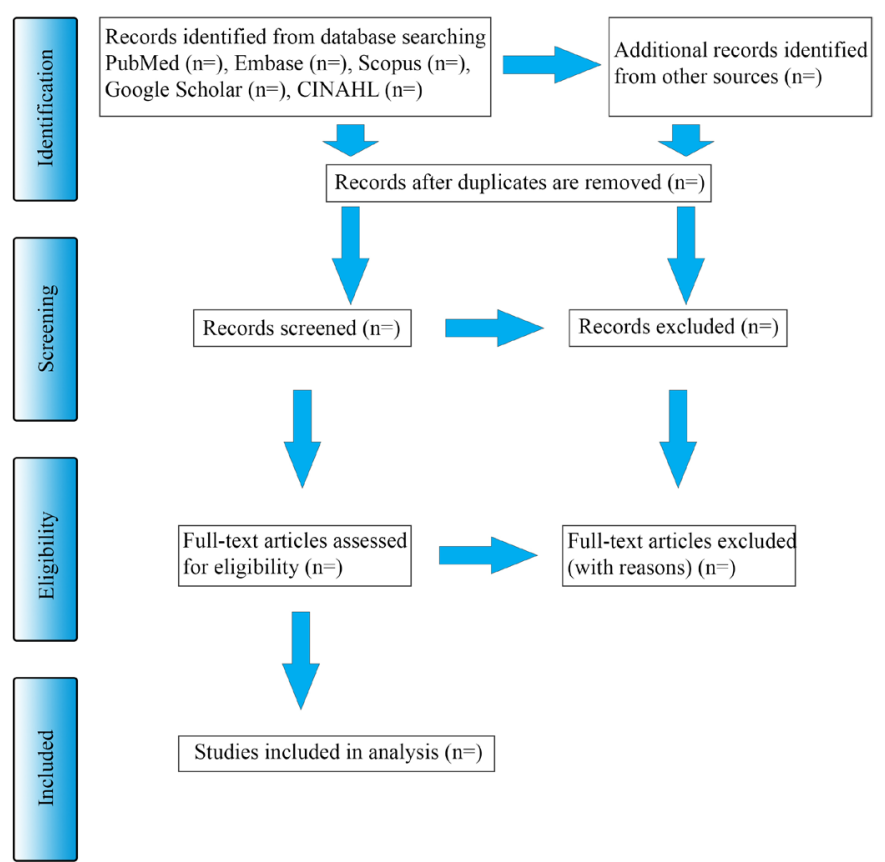

Figure 1 Preferred Reporting Items for Systematic Reviews and Meta-Analyses (PRISMA) chart detailing the study selection process.

2' in the title or abstract. These inclusion criteria reduced the number of retained publications to 172 . After consultation with the research team, we agreed that this limited scope reduced the number of publications for review to a manageable amount, while also ensuring that we could answer our research questions if we modified them to focus solely on the COVID-19 pandemic.

Title and abstract screening was conducted by two researchers. Included articles were imported into Mendeley for full-article screening by five researchers. Any discrepancies were discussed among the researchers until a consensus was reached.

\section{Stage 4: charting the data}

After searching the databases, all identified citations were uploaded to Mendeley 1.19.4/2019 (Elsevier) and duplicates removed. Titles and abstracts of all articles were screened by two independent reviewers to determine if they met the study's inclusion criteria. Potentially relevant articles were reviewed in full against the inclusion criteria by two independent reviewers. Disagreements between the two reviewers at any stage were resolved through mutual discussion or, where necessary, consultation with a third reviewer. The results and study inclusion process will be presented on a PRISMA extension for Scoping Reviews flow chart ${ }^{22}$ (figure 1).

We will use a targeted rule set to extract 12 relevant pieces of data from the included articles. This data extraction table will be developed in accordance with the objectives of our scoping review, as well as domainspecific expertise from members of our research team to ensure that we identify all relevant information. The data extracted from all relevant documents will include the following: (1) author(s), (2) year of publication, (3) type of document, (4) aim or study purpose, (5) methodology, (6) countries or regions studied, (7) type(s) of digital surveillance technology studied, (8) how the technology under study is used for disease surveillance, (9) target population(s), (10) key findings, (11) outcomes, and (12) implications of technology use (eg, ethical, political, etc). Five researchers will pilot the data extraction table on five articles and then discuss the findings to determine whether adjustments need to be made.

\section{Stage 5: collating, summarising and reporting the results}

In line with our objective of mapping the breadth and depth of the literature, we will conduct a thematic analysis of the data extracted from the articles with the goal of identifying what kinds of studies of digital health surveillance technologies have been conducted; which technologies, countries and surveillance implications have been studied; what debates, discussions and tensions have emerged within the literature; and, where applicable, what gaps exist in the literature. The analysis will be undertaken as a collective effort among our team of researchers to ensure an interdisciplinary analysis from multiple expert perspectives.

\section{PATIENT AND PUBLIC INVOLVEMENT}

Patients and the public were not involved in this research in any way.

\section{DISCUSSION}

The aim of this scoping review is to explore the literature on digital health surveillance technology, with the goal of mapping the research that has been done in this area, understanding the implications of use and highlighting any gaps. As digital health surveillance technologies are leveraged by countries around the world in an attempt to manage the COVID-19 pandemic, there is an urgent need to understand the potential short-term and longterm implications of technology use. We anticipate that the results of this scoping review will support informed decision-making around digital surveillance use and provide important insight into the existing knowledge of digital health surveillance technologies and the use of these forms of surveillance in monitoring and mitigating pandemics.

\section{ETHICS AND DISSEMINATION}

Given we are reviewing secondary sources and not working with human subjects, our scoping review did not require ethics approval. The findings of our scoping review will be disseminated through traditional academic channels, including peer-reviewed publications and conference presentations. We will also engage targeted public organisations through social media channels and accessible research briefs 
and infographics, developed with our interdisciplinary team of researchers. We will target our dissemination to global public health organisations. We will also target technology industry companies and community-based organisations dealing with the public response to the COVID-19 pandemic. Dissemination of our findings is intended to generate a shared understanding of the concept of digital surveillance, and to facilitate reflection and discussion on the benefits and challenges of pandemic surveillance strategies.

\section{Author affiliations}

${ }^{1}$ Arthur Labatt Family School of Nursing, Faculty of Health Sciences, Western University, London, Ontario, Canada

${ }^{2}$ Nursing, Fanshawe College, London, Ontario, Canada

${ }^{3}$ Faculty of Western Law, Western University, London, Ontario, Canada

${ }^{4}$ School of Health Studies, Facutly of Health Sciences, Western University, London, Ontario, Canada

${ }^{5}$ Geography and Environment, Faculty of Social Sciences, Western University, London, Ontario, Canada

${ }^{6}$ Department of Epidemiology and Biostatistics, Schulich School of Medicine and Dentistry, Western University, London, Ontario, Canada

${ }^{7}$ Department of Medicine, Schulich School of Medicine and Dentistry, Western University, London, Ontario, Canada

${ }^{8}$ The Africa Institute, Western University, London, Ontario, Canada

${ }^{9}$ Department of Population Health, Luxembourg Institute of Health, Strassen,

Luxembourg

${ }^{10}$ Faculty of Information and Media Sciences, Western University, London, Ontario, Canada

${ }^{11}$ Surveillance Studies Centre, Queen's University, Kingston, Ontario, Canada

${ }^{12}$ Department of Western Libraries, Western University, London, Ontario, Canada

\section{Twitter Brad Hiebert @bradhiebs}

Contributors LC and MN contributed to the acquisition, analysis and interpretation of data for the work, as well as drafting and contributing to revising the work for intellectual content. LD, JH, BH, JJS, MJS, JG, SS, AK, JB, TC, JL, JMS and DB contributed to the design of the study, interpretation of data and revising drafts for interdisciplinary intellectual content. MS contributed to developing the search strategy.

Funding This work was supported by the University of Western Ontario FHS Research Grant.

Competing interests None declared.

Patient consent for publication Not required.

Provenance and peer review Not commissioned; externally peer reviewed.

Supplemental material This content has been supplied by the author(s). It has not been vetted by BMJ Publishing Group Limited (BMJ) and may not have been peer-reviewed. Any opinions or recommendations discussed are solely those of the author(s) and are not endorsed by BMJ. BMJ disclaims all liability and responsibility arising from any reliance placed on the content. Where the content includes any translated material, BMJ does not warrant the accuracy and reliability of the translations (including but not limited to local regulations, clinical guidelines, terminology, drug names and drug dosages), and is not responsible for any error and/or omissions arising from translation and adaptation or otherwise.

Open access This is an open access article distributed in accordance with the Creative Commons Attribution Non Commercial (CC BY-NC 4.0) license, which permits others to distribute, remix, adapt, build upon this work non-commercially, and license their derivative works on different terms, provided the original work is properly cited, appropriate credit is given, any changes made indicated, and the use is non-commercial. See: http://creativecommons.org/licenses/by-nc/4.0/.

\section{ORCID iDs}

Anita Kothari http://orcid.org/0000-0003-1533-6976

Leigha Comer http://orcid.org/0000-0001-8848-8685
REFERENCES

1 Kind C. What will the first pandemic of the algorithmic age mean for data governance? Ada lovelace institute, 2020. Available: https:// www.adalovelaceinstitute.org/what-will-the-first-pandemic-of-thealgorithmic-age-mean-for-data-governance/ [Accessed 29 May 2021].

2 Bennet CJ, Haggerty KD, Lyon D. Transparent lives: surveillance in Canada. Edmonton, Alberta: Athabasca University Press, 2014.

3 Lyon D. Surveillance studies: an overview. Oxford: Polity Press, 2007.

4 Wang CJ, Ng CY, Brook RH. Response to COVID-19 in Taiwan: big data analytics, new technology, and proactive testing. JAMA 2020;323:1341-2.

5 Human Rights Watch [Internet]. Mobile location data and Covid-19: Q\&A. Available: https://www.hrw.org/news/2020/05/13/mobilelocation-data-and-covid-19-qa [Accessed 29 May 2021].

6 Bogart N. Canadian officials eye digital contact tracing amid surveillance, privacy concerns. CTV news. Available: https://www. ctvnews.ca/health/coronavirus/canadian-officials-eye-digitalcontact-tracing-amid-surveillance-privacy-concerns-1.4915845 [Accessed 28 Apr 2020].

7 Human Rights Watch [Internet]. Governments should respect rights in COVID-19 surveillance. Available: https://www.hrw.org/news/2020/ 04/02/governments-should-respect-rights-covid-19-surveillance [Accessed 29 May 2021].

8 Rowe F. Contact tracing apps and values dilemmas: a privacy paradox in a neo-liberal world. Int J Inf Manage 2020;55:102178.

9 Vitak J, Zimmer M. More than just privacy: using contextual integrity to evaluate the long-term risks from COVID-19 surveillance technologies. Soc Media Soc 2020;6:205630512094825.

10 Toh A, Brown D. How digital contact tracing for COVID-19 could worsen inequality. Human Rights Watch [Internet], 2020. Available: https://www.hrw.org/news/2020/06/04/how-digital-contact-tracingcovid-19-could-worsen-inequality [Accessed cited 2021 May 29].

11 Kennedy $\mathrm{H}$. Should more public trust in data-driven systems be the goal? ADA Lovelace Institute, 2020. https://www. adalovelaceinstitute.org/blog/should-more-public-trust-in-datadriven-systems-be-the-goal/

12 Owen T. Should we use digital contact tracing at all? Centre for International Governance Innovation [Internet] 2020 https://www. cigionline.org/articles/should-we-use-digital-contact-tracing-all (cited 2021 May 29).

13 Smith MJ, Axler R, Bean S, Rudzicz F, et al. Four equity considerations for the use of artificial intelligence in public health. Bull World Health Organ 2020;98:290-2.

14 Maati A, Švedkauskas Žilvinas, Svedkauskas Z. Framing the pandemic and the rise of the digital Surveilance state. Mezinárodni vztahy 2020;55:48-71.

15 Moerel L. Contact tracing apps: Why tech solutionism and privacy by design are not enough. International association of privacy professionals [Internet], 2020. Available: https://iapp.org/news/a/ contact-tracing-apps-why-tech-solutionism-and-privacy-by-designare-not-enough/ [Accessed 29 May 2021].

16 International Civil Liberties Monitoring Group [Internet]. Joint statement: digital surveillance technologies and COVID-19 in Canada. Available: https://iclmg.ca/digital-surveillance-covid-19/ [Accessed cited 2021 May 29].

17 Patel R. Removing the pump handle - stewarding data at times of public health emergency. Ada lovelace institute, 2020. Available: https://www.adalovelaceinstitute.org/removing-the-pump-handlestewarding-data-at-times-of-public-health-emergency/ [Accessed 29 May 2021].

18 Bowman C, Grindrod P. The technical and ethical distinction between random and non-random biometric data. Ada Lovelace Institute, 2019https://www.adalovelaceinstitute.org/the-technical-and-ethicaldistinction-between-random-and-non-random-biometric-data/

19 Wilson A. Context, agenda and ways of working, 2018. Available: https://www.adalovelaceinstitute.org/context-agenda-and-ways-ofworking/ [Accessed 29 May 2021].

20 Arksey H, O'Malley L. Scoping studies: towards a methodological framework. Int J Soc Res Methodol 2005;8:19-32.

21 Colquhoun HL, Levac D, O'Brien KK, et al. Scoping reviews: time for clarity in definition, methods, and reporting. J Clin Epidemiol 2014;67:1291-4.

22 Levac D, Colquhoun H, O'Brien KK. Scoping studies: advancing the methodology. Implement Sci 2010;5:69.

23 Peters MDJ, Godfrey C, Mclnerney PAromataris E, Munn Z, eds. Scoping reviews. JBI manual for evidence synthesis, 2020.

24 Moher D, Shamseer L, Clarke M, et al. Preferred reporting items for systematic review and meta-analysis protocols (PRISMA-P) 2015 statement. Syst Rev 2015;4:1. 